\title{
Enquête
}

Archives de la revue Enquête

$8 \mid 1993$

Varia

\section{Force et faiblesse des pactes littéraires}

Notes sur le jugement esthétique par défaut

Jacques Vialle

\section{(2) OpenEdition}

Journals

Édition électronique

URL : http://journals.openedition.org/enquete/180

DOI : 10.4000/enquete. 180

ISSN : 1953-809X

Éditeur :

Cercom, Éditions Parenthèses

Édition imprimée

Date de publication : 2 septembre 1993

Pagination : 139-157

Référence électronique

Jacques Vialle, "Force et faiblesse des pactes littéraires », Enquête [En ligne], 8 | 1993, mis en ligne le

09 juillet 2013, consulté le 19 avril 2019. URL : http://journals.openedition.org/enquete/180 ; DOI :

10.4000/enquete. 180

Ce document a été généré automatiquement le 19 avril 2019. 


\title{
Force et faiblesse des pactes littéraires
}

\author{
Notes sur le jugement esthétique par défaut
}

Jacques Vialle

1 En sociologie de la culture, la notion de goût tient généralement lieu de métaphore des partages sociaux; c'est le cas lorsque l'on parle du goût "plébéien» des classes populaires ou des "prétentions esthétiques" de la petite bourgeoisie. Cette façon d'évaluer les goûts, qui anticipe largement sur une théorie de l'ordre social, fait du jugement le plus singulier l'indice d'une situation symbolique qui l'excède en finalité (déclarer que l'on aime telle ou telle œuvre serait défendre avant tout une certaine idée de la légitimité culturelle). À tous égards, ce qui relie un jugement de goût à une œuvre n'apparaît pas comme une dimension de l'expérience esthétique : apprécier tel tableau de Kandinsky, tel poème de Mallarmé, renvoie le sociologue à un monde où les choses de l'art valent ce que valent leurs signes.

2 En s'obligeant ainsi à ne considérer que de très haut (ou de très loin) l'effet propre des œuvres, la sociologie des goûts fait l'économie d'une comptabilité embarrassante puisque, résumée à un titre, un nom d'auteur ou une catégorie générique, l'œuvre littéraire ou l'œuvre d'art ne se présente plus à ses instruments comme une structure sémiotique complexe et active, mais comme un symbole que l'on peut disposer sur un plan factoriel aux côtés d'autres symboles résumant eux-mêmes toutes sortes d'attributs (position sociale, niveau d'étude, classe d'âge, sexe, etc.).

3 Les difficultés que l'on rencontre à vouloir se saisir empiriquement du contenu d'une expérience esthétique sont de deux sortes :

- en premier lieu, nous ne savons établir de lien causal précis entre une œuvre, en tant que structure sémiotique, et les effets qu'elle produit;

- en second lieu, le langage de l'appréciation, qui relie l'œuvre à l'expérience esthétique, est vide de contenu descriptif: dire d'une œuvre qu'elle est "belle», "équilibrée », « harmonieuse », ou affirmer le contraire ne nous apprend rien sur elle. Les prédicats du jugement de goût indiquent seulement la coloration positive ou négative de l'appréciation; hormis cela, leur emploi ressort d'une forme de langage privé. Autrement 
dit, deux individus peuvent employer le même prédicat pour qualifier une même œuvre, sans que l'on puisse affirmer que ces jugements recouvrent la même somme de sensations.

4 En présentant ici les premiers résultats d'une enquête sur la réception de textes littéraires, nous montrerons que le jugement de goût n'est pas une instance aussi indécomposable qu'il paraît, du moment que l'on concentre les moyens de l'observation sur la situation élémentaire où des individus, identifiés à certaines de leurs propriétés, doivent se prononcer sur des œuvres, par ailleurs, également identifiables.

5 L'enquête dont il s'agit porte sur un échantillon stratifié de quatre-vingt-un lecteurs auxquels nous avons soumis quinze extraits d'œuvres littéraires présentés sans titre ni nom d'auteur et que chacun devait juger en fonction d'une grille standard d'appréciation.

6 Entre autres consignes, nous demandions au lecteur d'évaluer le plaisir pris à la lecture de chaque texte sur une échelle numérique standard: sept notes, ordonnées entre 0 et 6 , avaient valeur d'opinion.

7 Une note étant nécessairement supérieure, inférieure, ou égale à une autre, ce mode d'appréciation des œuvres introduisait une relation d'ordre entre les opinions d'un même lecteur, si bien que toute note attribuée pouvait être considérée comme l'expression d'une préférence sur un ensemble d'objets. Pour chaque lecteur, nous disposions ainsi d'un préordre de préférence sur quinze textes ${ }^{1}$.

8 L'avantage d'une représentation ordinale des notes sur une prise en compte plus directe de leur valeur cardinale était de gommer les fluctuations, à la hausse ou à la baisse, des notations interindividuelles: au moment de comparer les scores moyens obtenus par chaque texte, la sévérité de certains lecteurs ne se voyait pas, de cette façon, contaminée par la retenue de certains autres pour, en fin de compte, donner un résultat de compromis qui n'aurait pas exprimé le résultat collectif recherché. Nous retrouvions là, l'exigence méthodologique qui, depuis Condorcet et son Essai sur l'analyse de la probabilité des décisions rendues à la pluralité des voix [1785], fonde toutes les procédures de décision visant à déterminer le classement majoritaire d'un ensemble de candidats ayant subi l'épreuve d'un vote ou le sort d'une confrontation sportive. La règle majoritaire de Condorcet, dans le cas d'une assemblée de votants ayant exprimé son opinion sous la forme d'un ensemble d'ordres ou de préordres de préférence, détermine, pour chaque paire de candidats évalués, le candidat préféré et la position qu'il occupe dans l'ordre de préférence majoritaire.

9 En dénombrant, pour toute paire de textes [Ti, Tj], le nombre de fois où Ti (resp. Tj) est préféré à $\mathrm{Tj}$ (resp. Ti), nous pouvions ainsi représenter l'ensemble des préordres de préférence sous la forme d'un tournoi, et calculer, à partir de là, l'ordre médian le plus représentatif de l'opinion collective, soit l'ordre total qui contredisait le moins de préférences individuelles (fig. 1)2. 
Fig. 1. Des notes individuelles aux préférences collectives (exemple d'une assemblée de 5 candidats devant évaluer les textes 10 à 15)

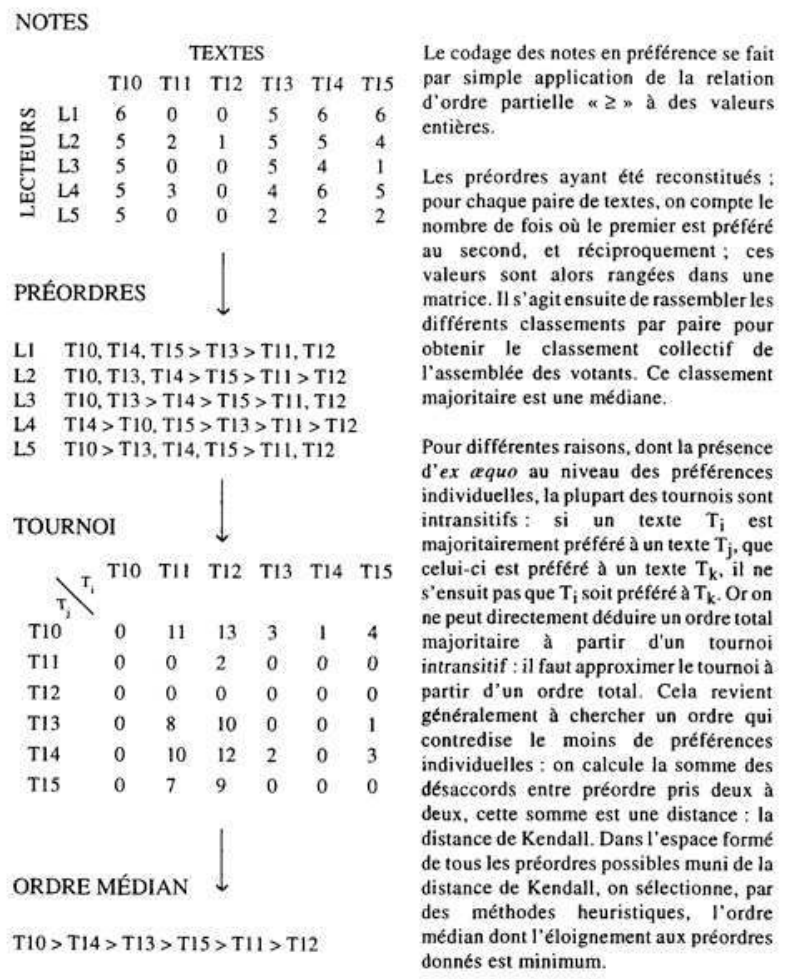

10 La taille et la composition de l'échantillon privilégiaient l'étude des effets du capital scolaire - indice classiquement retenu en sociologie de la culture - sur les préférences littéraires. Ayant préalablement défini trois niveaux de qualification scolaire, nous avons sélectionné trois groupes de lecteurs d'effectifs comparables ${ }^{3}$.

11 La superposition des ordres de préférence médians obtenus pour ces groupes de lecteurs nous permet de distinguer deux pôles d'opinions stables et une large zone de variation (fig. 2.1). Les textes 10 et 14 obtiennent un suffrage maximum, quel que soit le niveau d'étude de leurs lecteurs, alors que les textes 11 et 12 se voient préférer tous les autres.

$12 \mathrm{Au}$ regard de la mobilité des préférences intermédiaires, cette stabilité des opinions extrêmes se détache comme un fait véritablement singulier. En toute logique, si l'on considère que les différences de niveau d'instruction, par lesquelles on cherche à déterminer une certaine variabilité comportementale, appliquent uniformément leurs effets à l'ensemble des textes soumis à l'appréciation, on doit également considérer que certaines qualités sont à l'œuvre dans les textes 10,14, 11 et 12 qui forcent l'accord collectif dans un sens comme dans l'autre. C'est dire que la recherche d'une interprétation concernant ces accords exige que l'on s'interroge sur les vertus intrinsèquement rassembleuses des textes 10,14,11 et 12 . 
Fig. 2.1. Ordres de préférence médians selon le niveau d'étude

\begin{tabular}{|c|c|c|c|c|c|c|c|c|c|c|c|c|}
\hline$\leq \mathrm{Bac}:$ & T15 & $\mathrm{T} 14$ & T10 & $T 2$ & $\mathrm{~T} 13$ & T4 & T9 & T1 & $\mathrm{T} 7$ & $\mathrm{~T} 3$ & T6 & $\mathrm{T} 12 \mathrm{~T} 11$ \\
\hline$\langle$ Baclà 3$\rangle$ : & T14 & T10 & $\mathrm{T} 4$ & $\mathrm{~T} 7$ & T15 & $\mathrm{T} 2$ & T9 & $\mathrm{TI}$ & $\mathrm{T} 13$ & $\mathrm{T3}$ & T5 & $\mathrm{T} 11 \quad \mathrm{~T} 12$ \\
\hline$\geq \mathrm{Bac}+4$ : & T14 & $\mathrm{T} 4$ & T10 & $\mathrm{T7}$ & T9 & $\mathrm{T} 15$ & $\mathrm{~T} 8$ & $\mathrm{~T} 2$ & $\mathrm{~T} 3$ & T6 & $\mathrm{T} 13$ & $\mathrm{~T} 11 \quad \mathrm{~T} 12$ \\
\hline
\end{tabular}
$(10,14)$ et $(11,12)$, que nous formulerons provisoirement en termes de distance narratologique : les textes 10 et 14 donnent directement à voir ce dont ils traitent; les personnages, comme les situations, sont clairement identifiables, et les valeurs mises en jeu sont, pour ainsi dire, universelles. Les textes 11 et 12, quant à eux, brouillent les cartes de la représentation fictionnelle au moyen de divers procédés d'écriture et de composition; le sens, i.e. l'intention expressive, n'y est délivré qu'au prix d'une rupture avec certaines habitudes de lecture ${ }^{4}$.

19 Si le succès des textes 10 et 14 n'offre ici aucune prise sérieuse à conjecture, le rejet majoritaire des textes 11 et 12 sera envisagé, dans ce qui suit, comme le symptôme d'un échec ou d'une résistance des lecteurs à entrer dans un pacte littéraire dont ils ne distinguent pas clairement les règles. 
Compte tenu du caractère technique de celles-ci, on pouvait s'attendre à voir l'effet des résistances ou des échecs à la réception diminuer avec l'élévation du niveau de diplôme des lecteurs. Ce phénomène qui, nous l'avons vu, n'affecte pas de façon sensible les préférences littéraires, semble influencer plus directement le comportement des notes : les courbes de fréquences cumulées à chaque seuil montrent, pour les textes 11 et 12, une notation d'autant plus généreuse que le niveau scolaire du lectorat augmente (fig. 2.2). Le phénomène s'observe également à propos du texte 8, extrait d'Ulysse de James Joyce qui présente lui aussi certaines particularités formelles - dont l'usage systématique du monologue intérieur. Ici aussi, la comparaison des courbes de fréquences cumulées à chaque seuil révèle, chez les détenteurs de titres universitaires, une plus grande tolérance à certains procédés non usuels de la fiction (fig. 2.2).

Fig. 2.2. Diagrammes des fréquences de notes accordées aux textes 11,12 et 8 selon les niveaux de diplôme

Texte 11

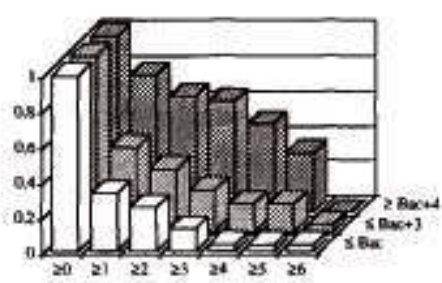

Texte 12

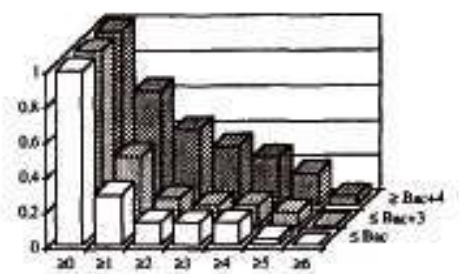

Texte 8

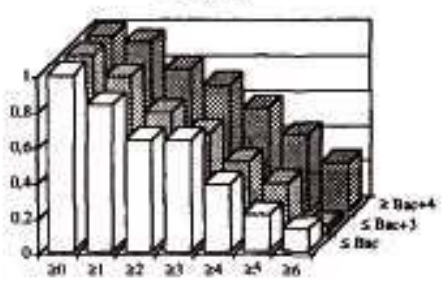

Chaque barre de diagramme représente la fréquence (notée en ordonnée) des notes supérieures ou égales à la valeur indiquée en abscisse. Le cumul des fréquences, à chaque seuil, donne aux diagrammes leur allure décroissante : plus rapide est cette décroissance, plus sévère est l'évaluation.

Pour autant, le capital scolaire n'est pas un indice si mécaniquement sensible aux partages culturels que l'on doive s'en contenter toujours et partout. C'est même une chance de constater ici la faiblesse de son pouvoir discriminant lorsque l'on passe des notes aux préférences, car on ne saurait trop se méfier de ce sésame de l'interprétation sociologique. La notion de capital scolaire est un pis-aller conceptuel qui présente l'avantage d'une manipulation aisée mais dont on mésestime généralement le pouvoir d'arrêt sur le raisonnement sociologique. Sous ce concept se cache, en effet, ce que les logiciens appellent un prédicat « composite » qui, comme son nom l'indique, recouvre un ensemble d'attributs hétérogènes. Il suffit de songer à toutes les variétés de compétences générales et particulières que sélectionne l'apprentissage scolaire pour mesurer combien pauvre est l'effet de connaissance résultant du simple constat d'une distribution inégale des appréciations selon le niveau de diplôme. Il n'est pas besoin d'être nominaliste pour arriver à cette conclusion que le genre de propriété que l'on entend désigner par le terme 
de capital scolaire n'a d'existence que métaphorique ; l'imagination sociologique devrait suffire à ne pas trop confondre ici les noms et les choses. transition, suppose plus que l'effet d'une juste rétribution des efforts investis au long d'une scolarité ; la tolérance esthétique et la souplesse mentale que requiert cette sorte de gymnastique ne vont pas sans une certaine ouverture à la création littéraire et aux quelques-uns de ses produits que l'édition parvient à diffuser. Pour caractériser cette attitude d'ouverture, dont on peut supposer qu'elle fournit le principe d'une meilleure explication des faits représentés fig. 2.2, nous avons songé à mesurer le genre d'inflexions qu'elle pouvait opérer sur les consommations littéraires. En remontant des choix de lecture signifiés par les titres d'ouvrages que les lecteurs déclaraient avoir lus le plus récemment, au type d'investissement culturel que supposent ces choix, en vertu du volume de circulation et du caractère plus ou moins atypique des œuvres auxquelles ils renvoient, nous pensions tenir un indice concret de l'attitude culturelle mise en cause dans notre raisonnement.

À l'image de ce que l'on observe en bourse, on peut supposer que le marché littéraire produit lui aussi ses risques à l'investissement en fonction du degré de consensus qui s'établit autour des œuvres: un roman dont le tirage atteint rapidement cent mille exemplaires, qui reçoit tel ou tel prix littéraire ou bénéficie de tel ou tel succès critique, est un produit culturel qui se présente cerné de garanties, et cette condition suffit à la plupart des lecteurs pour se déterminer, même si elle ne garantit pas ipso facto tout le bénéfice de leur choix. Inversement, les œuvres dont le tirage et la diffusion sont faibles offrent un accès plus délicat, parfois sans garantie, exigeant de leurs lecteurs une certaine disposition à l'investissement à risque. À volume de lecture et à niveau d'étude comparables, deux lecteurs peuvent encore, selon cette conception, se distinguer radicalement par leur attitude vis-à-vis de la production littéraire et, par extension, vis-àvis des œuvres elles-mêmes; c'est ce que nous avons cherché à caractériser au moyen d'un indicateur empirique de l'investissement culturel en matière de lecture. Ayant pris soin de recenser les livres lus par nos lecteurs au cours des trois derniers mois précédant l'enquête, nous disposions de l'information nécessaire à la mise en œuvre de cette opération ${ }^{5}$.

cités ont été indexés selon trois caractéristiques: une caractéristique de forme qui, chez nous, oppose le récit traditionnel aux fictions dites "pures»; une caractéristique d'époque qui indique la longévité d'une œuvre sur le marché littéraire et que nous avons divisée en trois classes : moins de cinq ans, moins de trente ans ou plus de trente ans ; le volume de ventes, enfin, qui symbolise le niveau de circulation de l'œuvre et dont nous avons distingué trois seuils : plus de quarante mille exemplaires, moins de quarante mille et moins de cinq mille ${ }^{6}$.

De cette façon, chaque œuvre a pu être représentée comme une combinaison de trois modalités. Chaque combinaison a été affectée d'une valeur d'investissement culturel: « fort », « moyen » ou « faible » (fig. 3). 
Fig. 3. Codage des titres

\begin{tabular}{|c|c|c|c|c|}
\hline Genre & Époque & Tirage & Investissement & Fréquence \\
\hline Fiction traditionnelle & - de 5 ans & plus de 40000 ex & Faible & 0.254 \\
\hline Fiction traditionnelle & - de 5 ans & de 5000 a $40000 \mathrm{ex}$. & Moyen & 0.028 \\
\hline Fiction traditionnelle & - de 5 ans & moins de $5000 \mathrm{ex}$. & Fort & 0.011 \\
\hline Fiction traditionnelle & entre 5 et 30 ans & plus de 40000 ex & Faible & 0.275 \\
\hline Fiction traditionnelle & entre 5 et 30 ans & de 5000 a 40000 ex. & Moyen & 0.039 \\
\hline Fiction traditionnelle & entre 5 et 30 ans & moins de 5000 ex. & Fort & 0.002 \\
\hline Fiction traditionnelle & au-delà de 30 ans & plus de $40000 \mathrm{ex}$. & Faible & 0.313 \\
\hline Fiction traditionnelle & au-dela de 30 ans & de 5000 a 40000 ex. & Moyen & 0.014 \\
\hline Fiction traditionnelle & au-delà de 30 ans & moins de 5000 ex. & Fort & 0.001 \\
\hline Fiction " pure " & - de 5 ans & plus de $40000 \mathrm{ex}$. & Moyen & 0.006 \\
\hline Fiction " pure " & - de 5 ans & de 5000 d 40000 ex. & Fort & 0.003 \\
\hline Fiction a pure " & - de 5 ans & moins de $5000 \mathrm{ex}$. & Fort & 0.001 \\
\hline Fiction " pure " & entre $5 \mathrm{et} 30$ ans & plus de 40000 ex. & Moyen & 0.022 \\
\hline Fiction * pure " & entre 5 et 30 ans & de 5000 a 40000 ex. & Fort & 0.001 \\
\hline Fiction " pure - & entre 5 et 30 ans & moins de $5000 \mathrm{ex}$. & For & 0.003 \\
\hline Fiction * pure " & au-delà de 30 ans & plus de 40000 ex. & Moyen & 0.021 \\
\hline Fiction = pure " & au-delà de 30 ans & de 5000 a 40000 ex. & Fort & 0.001 \\
\hline Fiction " pure * & au-dela de 30 ans & moins de $5000 \mathrm{ex}$. & Fort & 0.004 \\
\hline
\end{tabular}

Pour donner un aperçu de la répartition des différents items du codage sur l'ensemble des titres recueillis, nous avons fait figurer leur fréquence d'occurrence. Remarquons que ces fréquences décrivent une échelle de la rareté qui se trouve correspondre terme-à-terme à l'échelle des investissements. Cette correspondance, que l'on pouvait espérer mais non pas prévoir, vérifie, sur un vaste ensemble d'objets, la cohérence de notre indexation.

La dernière étape du traitement consistait à regrouper les lecteurs qui, dans leur consommation littéraire, témoignaient d'au moins deux investissements forts ou de trois investissements moyens.

L'ordre de préférence médian obtenu sur le groupe ainsi isolé fait apparaître une progression considérable des textes 8,11 et 12 ; par ailleurs, plus de cinquante pour cent des notes accordées au texte 11 sont supérieures ou égales à cinq, contre un peu moins de quarante pour cent de celles accordées au texte 12 (fig. 4.1 et 4.2).

Les textes 10 et 14, quant à eux, se maintiennent parmi les leaders, ce qui laisse penser que le consensus invariable dont ils font l'objet recouvre un ensemble de valeurs esthétiques, psychologiques ou affectives peu sensibles aux oppositions culturelles que la stratification sociale de notre lectorat laisse pourtant supposer.

Le pouvoir discriminant de notre indicateur tranche d'autant plus nettement sur celui de la variable scolaire que l'on compare l'ordre de préférence médian des «forts investisseurs » à celui du reste des lecteurs que, faute de mieux, nous nommons « faibles investisseurs " (fig. 4.1). Quant au niveau d'interdépendance de ces deux modes de regroupement - le capital scolaire et les dispositions à l'investissement littéraire - on constate que le premier n'englobe pas le second à titre d'élément, mais qu'il existe entre eux une certaine "viscosité »: malgré soixante-dix-huit pour cent d'individus dont le niveau de diplôme est supérieur ou égal à «Bac +4 ", les «forts investisseurs " ne 
représentent, à l'échelle de l'échantillon, que quarante-quatre pour cent de cette catégorie (fig. 4.3).

31 Autrement dit, s'il semble nécessaire de disposer d'un fort capital scolaire pour accéder à un certain niveau de consommation littéraire et jouir des dispositions culturelles qui y sont spécifiquement associées, cette seule condition ne suffit pas à décider de cet accès. Des comportements spécifiques, auxquels prédispose sans aucun doute un long apprentissage scolaire, doivent prendre le relais, qui contribuent à former le « sens critique » du lecteur, lequel est, pour partie au moins, un « savoir lire».

Fig. 4.1. Ordres de préférence médians selon le niveau d'étude (rappel) et le niveau d'investissement culturel

\begin{tabular}{|c|c|c|c|c|c|c|c|c|c|c|c|c|c|c|c|}
\hline$\leq \mathrm{Bac}:$ & T15 & T14 & $\mathrm{T} 10$ & $\mathrm{~T} 2$ & $\mathrm{~T} 13$ & $\mathrm{~T} 4$ & 19 & TI & $\mathrm{T} 7$ & T3 & T8 & T6 & TS & T12 & T11 \\
\hline$\langle$ Bacla3 $\rangle$ & T14 & T10 & $\mathrm{T} 4$ & $T 7$ & T15 & $\mathrm{T} 2$ & T9 & TI & $\mathrm{T} 13$ & $\mathrm{~T} 3$ & T8 & T5 & T6 & T11 & T12 \\
\hline$\geq \mathrm{Bac}+4:$ & T14 & T4 & T10 & $\mathrm{T} 7$ & $\mathrm{~T} 9$ & T15 & T8 & $\mathrm{T} 2$ & $\mathrm{~T} 3$ & T6 & TS & T13 & $\mathrm{T!}$ & TII & T12 \\
\hline & $:$ T14 & T10 & T15 & T4 & T9 & $T 7$ & $T 2$ & T13 & $\mathrm{Tl}$ & T3 & T6 & T5 & T8 & T11 & $\mathrm{T} 12$ \\
\hline Inv, fort. & $\mathrm{T} 8$ & T4 & $\mathrm{T} 14$ & $\mathrm{~T} 2$ & $\mathrm{~T} 10$ & T11 & T3 & T7 & $\mathrm{T} 12$ & T9 & T15 & T5 & T6 & $\mathrm{T} 13$ & $\mathrm{~T} 1$ \\
\hline
\end{tabular}

Fig. 4.2. Fréquences cumulées des notes au seuil de chacune d'entre elles

Texte 11

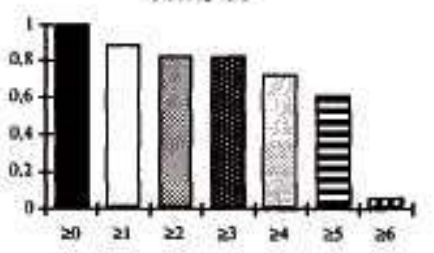

Texte 12

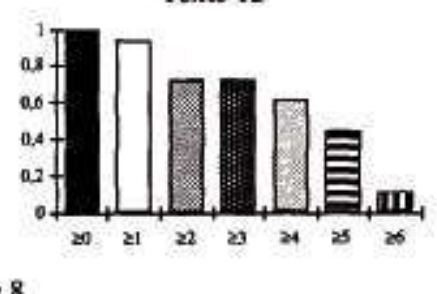

Texte 8

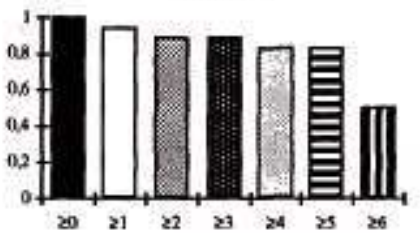

Fig. 4.3. Composition de la population des « forts investisseurs »

\begin{tabular}{l|cccc} 
& $\leq \mathrm{Bac}$ & {$[\mathrm{Bac}+1, \mathrm{Bac}+3]$} & $\geq \mathrm{Bac}+4$ & Total \\
\hline Effectifs & 1 & 3 & 14 & 18 \\
\% de la cat. & 4 & 12 & $\mathbf{4 4}$ & 22 \\
\% du gpe. & 5.5 & 16.5 & $\mathbf{7 8}$ & 100
\end{tabular}

Si $78 \%$ des « forts investisseurs » ont un niveau d'étude $\geq$ Bac +4 , moins de la moitié des personnes ayant acquis ce niveau d'instruction sont de «forts investisseurs ».

Pour confirmer cette autonomie relative de la variable "niveau d'investissement littéraire ", il nous restait à estimer, pour ainsi dire, " hors raisonnement sociologique ", la précision des traitements ayant conduit à isoler la fraction des « forts investisseurs » de l'ensemble des lecteurs. La question restait en effet de savoir jusqu'à quel point cette partition empirique de l'échantillon recoupait une partition objective des appréciations. 

de préférence individuels, l'analyse hiérarchique descendante présentait cet avantage de fractionner notre échantillon de départ en classes d'individus aux comportements semblables, sans autre hypothèse que mathématique. Tout l'intérêt d'appliquer cette méthode était alors de voir si certains fractionnements opérés par l'analyse hiérarchique correspondaient à la possession de telle ou telle propriété sociologiquement remarquable.

Le premier niveau de partition réalisé distingue deux groupes d'effectifs très inégaux $\left(\mathrm{N}_{1}\right.$ $=63$ et $\mathrm{N}_{2}=18$ ). Le groupe de moindre taille se trouve représenter, à lui seul, soixantedouze pour cent de l'effectif des «forts investisseurs» que nous avions isolés précédemment, ce qui offre une justification des plus solides au raisonnement sociologique par lequel nous étions amenés à opérer empiriquement cette partition. Le premier groupe $\left(\mathrm{N}_{1}\right)$, quant à lui, se compose de deux sous-ensembles de lecteurs $\left(\mathrm{N}_{3}=39\right.$ et $\mathrm{N}_{4}=24$ ), dont la composition nous éclaire davantage sur l'effet diffus du capital scolaire : $\mathrm{N}_{3}$ et $\mathrm{N}_{4}$ partagent sensiblement, mais non mécaniquement, les titulaires d'un diplôme universitaire moyen (entre bac +1 et bac +3 ) des lecteurs n'ayant pas atteint ce niveau (fig. 5).

Fig. 5. Produits des deux premiers niveaux de partition obtenus par analyse hiérarchique descendante (cf. aussi Annexe infra)

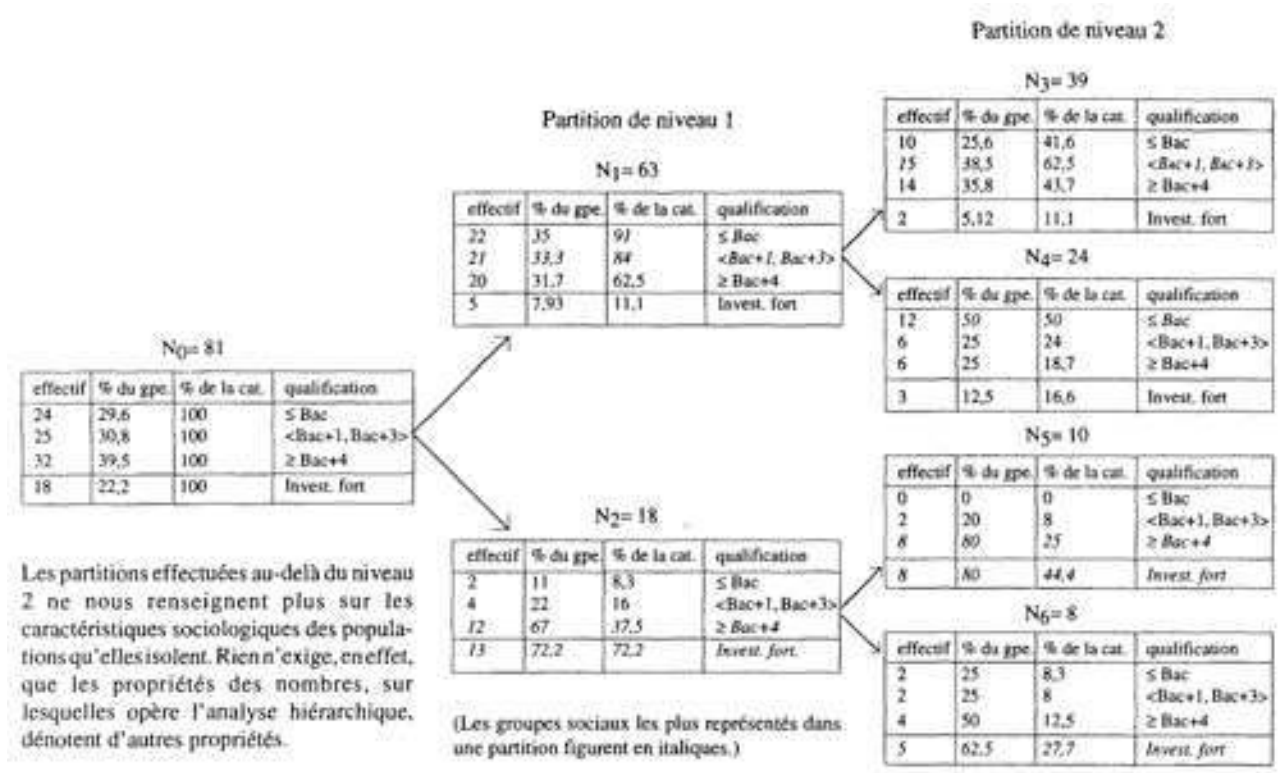

Nombreux sont les philosophes qui, depuis Frege, ont tenté de définir le type d'acte mental auquel nous contraignait la fiction, au regard des mécanismes ordinaires de la référence et de la prédication. Parmi eux, Searle est sans doute celui auquel nous devons la formulation la plus claire du statut logique des énoncés littéraires : «Ce sont, dit-il, des actes illocutoires feints [qui empruntent la forme] de réels actes d'énonciation [...] Les illocutions feintes qui constituent une œuvre de fiction, poursuit-il, sont rendues possibles par l'existence d'un ensemble de conventions qui suspendent l'opération normale des règles reliant les actes illocutoires et le monde ${ }^{7}$. » Entrer dans un pacte littéraire reviendrait, selon Searle, à accepter de faire comme si les énoncés de fiction étaient des énoncés sérieux tout en sachant qu'ils ne le sont pas : pour avoir, par exemple, cette impression qu'un texte «imite» ou « exprime» la réalité, le lecteur devra passer 
outre certains artifices d'écriture et feindre de croire au monde qu'on lui décrit sans en être embarrassé. C'est le jeu de l'auteur que de fournir le cadre bien construit de cette illusion, mais pour cela il doit compter sur la souplesse épistémologique de ses lecteurs ; un pacte de réception littéraire se présenterait ainsi comme un accord sur les conditions de l'illusion littéraire. Les scores d'appréciation des textes 11,12 et 8 , étudiés précédemment, montrent jusqu'à quel point se maintient la tolérance des lecteurs à certaines exigences formelles de la fiction : face à un texte dont la règle du jeu narratif leur est étrangère, ils ont toute chance de ne percevoir qu'un flux incohérent de paroles et de juger l'œuvre selon un canon auquel elle ne répond pas.

Dans Langages de l'art, Nelson Goodman développe une conception du plaisir pris aux œuvres qui insiste sur le nécessaire fonctionnement d'un arrière-plan de connaissance : «Puisque, dit-il, l'exercice, l'entraînement et le développement de nos pouvoirs de discriminer entre des œuvres sont des activités manifestement esthétiques, les propriétés esthétiques d'une [œuvre] n'englobent pas seulement celles qu'on trouve en la [consultant], mais également celles qui déterminent comment on doit la [consulter] ${ }^{8}$. "

Si nous ne jugeons pas un "polar » comme nous jugeons un poème, c'est bien, comme le suggère Goodman, qu'il est nécessaire qu'une chose nous apparaisse comme étant une certaine chose et non une autre pour que nous la jugions comme un exemplaire plus ou moins réussi et plus ou moins plaisant de cette sorte de chose.

Pour celui qui n'est pas familier de telle ou telle technique littéraire rencontrée au hasard d'une lecture, il ne sert sans doute à rien de consulter le Dictionnaire des littératures; pour lire à plein rendement le passage incriminé, il lui faut disposer d'un capital d'expérience tel que, aussitôt perçu, le trait en question lui apparaisse comme un fait de style plutôt que comme l'expression d'une pure fantaisie ; autrement dit, il doit percevoir ce trait comme un procédé conforme à ce qu'il est possible d'admettre en matière de fiction.

Les qualités esthétiques qu'une œuvre nous semble avoir ne dépendent donc pas seulement des propriétés esthétiques que nous percevons en elles; elles varient également selon que celles-ci sont perçues comme standards, variables ou contrestandards pour nous'.

Lorsqu'une œuvre fait violence à nos catégories de perception parce qu'elle présente certains traits non standards pour nous, l'effet d'étrangeté ressenti peut conduire à une rupture de l'effet esthétique. L'appréciation d'une œuvre "par défaut» nous semble épuiser entièrement cette conception des choses $^{10}$.

Le véritable jugement esthétique suppose une compréhension minimum de «ce qu'est l'œuvre ", soit une compréhension de son intention expressive puisqu'il sanctionne la réalisation plus ou moins réussie de cette intention. Mais, pour comprendre «ce qu'est une œuvre", encore faut-il qu'elle soit perçue comme l'extension valide d'un savoir "comment sont les œuvres ${ }^{11}$ ». La "bonne volonté culturelle», qui se mesure à l'éclectisme des expériences et au risque des goûts, n'est pas autre chose qu'une disposition générale à étendre facilement le domaine d'un savoir "comment sont les œuvres ", puisque c'est en accordant plus facilement une pertinence esthétique à certains effets d'étrangeté que l'on minimise au mieux les décalages perceptifs qu'ils produisent. Mais cette bonne volonté porte mal son nom puisque, n'étant pas de l'ordre d'un vouloir, elle n'est pas proprement intentionnelle.

La recherche d'indices permettant de discriminer plus finement entre des comportements de lecture, d'écoute musicale, ou de perception artistique, est une tâche 
qui s'impose à la sociologie des goûts du moment qu'elle cherche à s'augmenter d'une sociologie de la réception des œuvres. Cette recherche a ceci de particulier qu'elle se nourrit davantage de conjectures sur les mécanismes mentaux requis par telle ou telle activité esthétique, que de considérations tranchées sur les mécanismes sociaux que serviraient aveuglément ces activités. Pour autant, il ne s'agit pas de tenter une interprétation concurrente des mêmes faits. La question se pose seulement de savoir reconnaitre que, bien avant de faire valoir ses gains sur un marché de la distinction sociale, le plaisir de lire, comme tant d'autres plaisirs, n'a pour condition que de savoir être pris.

\section{BIBLIOGRAPHIE}

Les textes $8,10,11,12$ et 14 sont respectivement extraits de :

JoYCE J. - Ulysse, Paris, Gallimard, 1948, p. 54-59 (1 ${ }^{\text {re }}$ éd. Paris, 1922).

CARDINAL M. - Les Mots pour le dire, Paris, France-Loisirs, 1977, p. 41-44.

SochABA D. - « Palimpseste 9732 », Marseille, AGONE, 1, 1991, p. 71-77.

BecketT S. - Comment c'est, Paris, Minuit, 1961, p. 98-101.

GRUMBERG J.-C. - Zone libre, Arles, Actes Sud, 1990, p. 41-44.

Pour le reste, cf. :

BOURdieu P. - Les Règles de l'art. Paris, Seuil, 1992.

DegenNe A. - Techniques ordinales en analyse des données, Paris, Hachette, 1972.

DUCHET C. - « Comptes pour contes : réflexions sur la bibliométrie littéraire », in A. Vaillant, ed., Mesure(s) du livre, Paris, Bibliothèque nationale, 1992, p. 99-105.

Goodman N. - Langages de l'art, Nîmes, Jacqueline Chambon, 1990 (1 ${ }^{\text {re }}$ éd. Indianapolis, 1968).

GUÉNOCHE A. - «Order at minimum distance of a valued tournament », Table ronde Modélisation, analyse et agrégation des préférences et des choix, Marseille, 1988.

LyONS M. Le Triomphe du livre, Paris, Promodis, 1987.

MARTIN H.-J. - « Comment mesurer un succès littéraire. Le problème des tirages », in G. Barber, J. Petit \& R. Laufer, eds, La Bibliographie matérielle, Paris, Éd. du CNRS, 1983, p. 25-42.

MontJARDET B. - « Tournois et ordres médians pour une opinion », Math. Sci. hum., 43, 1973, p. 55-70.

MONTJARDET B. - «Éléments pour une histoire de la médiane métrique », in J. Feldman, G. Lagneau \& B. Matalon, eds, Moyenne, médiane, centre. Histoire et usages. Paris, Éd. de l’EHESS, 1991, p. 45-62.

NoRA P. - « L'évolution du best-seller », Atlas Universalis des littératures, Paris, Universalis, 1992, p. 396-397.

ORECCHIONi P. - « Eugène Sue : mesure d'un succès », Europe, 643-644, 1982, p. 157-166. 
SEARLE J. - Sens et expression, Paris, Minuit, 1982 ( $1^{\text {re }}$ éd. Cambridge, 1979).

SeARLE J. - L'Intentionnalité. Essai de philosophie des états mentaux, Paris, Minuit, 1985 (1 ${ }^{\mathrm{re}}$ éd.

Cambridge, 1983).

SHUSTERMAN R. - Critique et poésie selon I. A. Richards. De la confiance positiviste au relativisme naissant, Thèse d'état, Université Bordeaux III, 1988, multigr.

VAillant A. - «L'un et le multiple, essais de modélisation bibliométrique », in Id., Mesure(s) du livre, Paris, Bibliothèque nationale, 1992, 191-204.

WALTON K. - « Catégories de l'art » (1970), repris in Esthétique et poétique, Paris, Seuil, 1992, 81-129.

\section{NOTES}

1. Un préordre est un ordre total sur des classes d'équivalence : celles que forment ici les ex œquo puisque sept valeurs doivent servir à ordonner quinze objets.

2. Sur les méthodes ordinales de traitement des opinions, cf. A. Degenne (1972) ; B. Montjardet (1973) et (1991) ; A. Guénoche (1988). Le traitement de nos données d'enquête a été effectué au moyen du logiciel «A. PREF » conçu et généreusement transmis par Alain Guénoche (CNRS, GRTC, Marseille).

3. Soit 24 individus dont le niveau de diplôme est inférieur ou égal au baccalauréat; 25 dont le niveau se situe entre bac +1 et $\mathrm{bac}+3 ; 32$ individus, enfin, ayant obtenu un diplôme de niveau supérieur ou égal à bac +4 .

4. Les références des textes $10,11, ! 2,14$, sont données en fin d'article.

5. Sur ce que l'on peut déduire de la circulation des œuvres, mesurée aux tirages, aux réimpressions et aux rééditions, cf. C. Duchet (1992) ; A. Vaillant (1992) ; P. Orecchioni (1982) ; M, Lyons (1987) ; H-J. Martin (1983); P. Nora (1992). Sur les logiques économiques du monde de l'édition, cf. P. Bourdieu (1992), § 3, première partie.

6. Les volumes de ventes sont repris de Livres Hebdo. Pour le codage qualitatif des œuvres, nous avons fait appel à un libraire, trois éditeurs, et force discussions.

7. J. Searle (1982), p. 110.

8. N. Goodman (1990), p. 146 (souligné par nous).

9. Sur ce sujet, $c f$. Walton K. [1970].

10. Ayant soumis différents poèmes à l'appréciation d'un groupe d'étudiants de première année d'un cycle scientifique, et ayant pris soin de présenter ces poèmes sans aucune référence, reproduisant en cela l'expérience de I. A. Richard, R. Shusterman montre que certains jugements ne peuvent s'expliquer que par un échec de l'expérience littéraire à atteindre ses conditions de satisfaction. Cet échec tient selon lui à la non-familiarité du lecteur au type d'œuvre proposé à son jugement : «Ils n'ont pas, dit Shusterman, lu assez d'œuvres pour avoir des préjugés, et, a fortiori, des préjugés techniques. En vérité, ils sont relativement désarmés devant le fait poétique et font souvent ce que l'on pourrait appeler une erreur pragmatique sur la fonction de la poésie. » R. Shusterman (1988), p. 604.

11. Dans L'Intentionalité, Searle introduit et développe le concept de «savoir comment sont les choses ", qu'il oppose à un "savoir ce que sont les choses ", pour rendre compte de l'ensemble des compétences, des savoir-faire et des catégories de perception qui sous-tendent nos actions les plus ordinaires tout en échappant à l'intentionalité. C'est en référence aux développements de Searle que nous reprenons ici le concept de « savoir comment ». 